\title{
A obesidade como consequência das alterações endócrino-metabólicas no idoso: uma revisão narrativa
}

Obesity as a consequence of endocrine-metabolic alterations in elderly individuals: a narrative review

La obesidad como consecuencia de los cambios endocrinos-metabólicos en los ancianos: una revisión narrativa

Hetielle Amanda Silva Veiga1, Mariana Pontes de Oliveira ${ }^{1 *}$, Karen Regina Santana ${ }^{1}$.

\section{RESUMO}

Objetivo: Discutir as alterações endócrino metabólicas próprias do idoso como causa da obesidade. Revisão bibliográfica: $\mathrm{O}$ hormônio do crescimento $(\mathrm{GH})$, estrógeno e testosterona reduzem no envelhecimento. $\mathrm{O}$ cortisol, devido ao maior estresse no idoso, encontra-se aumentado. Também ocorre tanta elevação do hormônio tireoestimulante (TSH) com menor resposta da glândula ao seu estímulo, como queda na produção de triiodotironina (T3) e tiroxina (T4). A insulina e glucagon têm sua secreção alterada, contribuindo para o aumento da glicemia, que é proporcional à idade. A diminuição da secreção de leptina e aumento da grelina ocorrem durante o envelhecimento. Assim, todas essas alterações hormonais contribuem para a obesidade neste público. Considerações finais: A obesidade nos idosos ocorre uma vez que o envelhecer traz mudanças na composição corporal em relação ao tecido muscular, gordura e alterações na produção e liberação de vários hormônios ligados ao metabolismo, além da modificação nos hábitos de vida, como o sedentarismo.

Palavras-chave: Obesidade, Idoso, Hormônios.

\begin{abstract}
Objective: To discuss the endocrine-metabolic changes in elderly individuals as a cause of obesity. Bibliographic review: The growth hormone $(\mathrm{GH})$, estrogen and testosterone hormones are reduced in aging. Cortisol is increased due to a greater stress level in the elderly individuals. It also occurs thyrostimulant hormone (TSH) elevation with lower gland response to its stimulus as well as a decrease in the production of triiodothyronine (T3) and thyroxine (T4). Insulin and glucagon have their secretion altered, contributing to the increase in blood glucose, which is proportional to age. The decreased secretion of leptin and increased ghrelin occur with aging. Therefore, all of these hormonal changes contribute to obesity in this demographic. Final considerations: Obesity in elderly individuals occurs once aging brings changes in body composition in relation to muscle tissue, fat and changes in the production and release of various hormones linked to metabolism, besides the modification in the habits, such as sedentarism.
\end{abstract}

Keywords: Obesity, Elderly, Hormones.

\section{RESUMEN}

Metas: Discutir los cambios metabólicos endocrinos característicos de los ancianos como causa de la obesidad. Revisión bibliográfica: Las hormonas hormona de crecimiento (GH), estrógeno y testosterona disminuyen con el envejecimiento. El cortisol, debido al mayor estrés en los ancianos, aumenta. También hay

${ }^{1}$ Centro Universitário Municipal de Franca (Uni-FACEF), Franca - SP. *E-mail: maripontes.med@gmail.com 
un aumento de hormona tiroestimulante (TSH) con una menor respuesta de la glándula a su estímulo, así como una disminución en la producción de triyodotironina (T3) y tiroxina (T4). La secreción de insulina y glucagón se altera, contribuyendo al aumento de la glucemia, que es proporcional a la edade. Con el envejecimiento se produce una disminución de la secreción de leptina y un aumento de grelina. Así, todos estos cambios hormonales contribuyen a la obesidad en este público. Consideraciones finales: La obesidad en los ancianos se produce cuando el envejecimiento trae consigo cambios en la composición corporal en relación al tejido muscular, la grasa y cambios en la producción y liberación de diversas hormonas ligadas al metabolismo, además de cambios en los hábitos de vida, como el sedentarismo.

Palabras clave: Obesidad, Ancianos, Hormonas.

\section{INTRODUÇÃO}

O envelhecimento do ser humano é um fenômeno fisiológico que ocorre em escala global e considerado um processo gradual, complexo e inevitável em que há redução da capacidade do organismo em lidar com o estresse do meio ambiente aumentando a probabilidade de morte. Assim, não possui um marcador biofisiológico, sendo definido mais por fatores legais do que biológicos, tendo como marco legal a idade, que nos países desenvolvidos são idosos os indivíduos acima dos 65 anos, já nos subdesenvolvidos, a partir de 60 anos (LOPES AC, 2016; FREITAS EV, et al., 2013).

Outra classificação é quanto à senescência, que se refere ao conjunto de alterações no corpo humano, as quais fazem parte de um processo fisiológico, e a senilidade, caracterizada por um processo patológico, o qual não é comum a todos os idosos nem normal da idade, mas que geralmente está associado ao declínio das funções dos órgãos e sistemas (SBGG, 2016). No Brasil, a expectativa de vida em 2018 chegou à média de 76,2 anos que, aliada à redução da taxa de natalidade, faz o número de idosos crescer no país (BRASIL, 2020).

Mudanças genéticas e bioquímicas decorrentes dessa fase culminam em danos celulares e teciduais, também há redução de tecido muscular e ósseo que alteram o metabolismo geral e da gordura corporal, a qual aumenta com a idade. Além disso, a fisiologia dos sistemas é alterada quando comparada a um organismo jovem, principalmente o sistema endócrino, foco deste trabalho (SGARBIERI VC e PACHECO MTB, 2017).

Em geral, a reserva funcional dos órgãos é reduzida e as alterações hormonais no envelhecimento levam ao aumento da prevalência de doenças endocrinológicas, como a obesidade. Essa, considerada como um dos mais importantes problemas de saúde pública, acontece de um desequilíbrio entre ingestão alimentar e gasto energético, levando a um acúmulo de gordura corporal, na maioria das vezes. Devido a mudanças fisiológicas na composição corporal, como o aumento da gordura visceral, diminuição da massa muscular e perda de altura por compressão dos corpos vertebrais, o Índice de Massa Corpórea (IMC), divisão do peso pela altura em $\mathrm{kg} / \mathrm{m}^{2}$, passa a ser menos preciso por não distinguir massas gordurosa e magra, inferindo que não há avaliação perfeita para o diagnóstico de obesidade nessa faixa etária (ABESO, 2016; ALBUQUERQUE ALY, et al., 2019; HOLLANDA BC, et al., 2020; BRASIL, 2020; SOUZA YP, et al., 2018).

Com o aumento da expectativa de vida e a transição nutricional (redução dos casos de desnutrição e ascensão de indivíduos com sobrepeso/obesidade), é importante ressaltar que condições que provocam alteração no perfil metabólico como redução da tolerância à glicose, aumento da resistência à insulina e dos perfis lipídicos, aumentam o risco de doenças cardiovasculares (DCV), distúrbios musculoesqueléticos, alguns tipos de câncer e mortalidade. Assim, entender a ação de determinados hormônios do eixo hipotálamohipófise, insulina, glucagon, grelina e leptina é fundamental para compreensão da correlação entre envelhecimento e obesidade (FREITAS EV, et al., 2013; JESUS AS e ROCHA SV, 2018; SOUZA YP, et al., 2018).

Mesmo sabendo que, com o envelhecimento, o tecido adiposo sofre mudanças em sua quantidade, distribuição, composição celular, resposta endócrina, inflamação e alteração na capacidade regenerativa, causando uma piora na qualidade de vida dos idosos obesos, muitos profissionais ainda não recomendam o 
emagrecimento para esses pacientes devido à falta de estudos que mostram seus reais benefícios (BEZERRA DS, et al., 2017; ALBUQUERQUE ALT, et al., 2020).

Sendo assim, este trabalho tem como objetivo discutir as alterações endócrino metabólicas próprias do idoso como causa da obesidade. Devido à necessidade da exploração do tema, carência de publicações acerca do assunto, sua atual relevância e aumento na prevalência da obesidade e da expectativa de vida, viu-se uma oportunidade para trazer estudos mais atuais sobre essa relação contribuindo à literatura acadêmica contemporânea.

\section{REVISÃO BIBLIOGRÁFICA}

$\mathrm{O} \mathrm{GH}$, produzido na adeno-hipófise, exerce seu efeito diretamente sobre os tecidos, aumenta a síntese proteica e a mobilização de ácidos graxos e reduz a utilização de glicose. A secreção se dá de maneira pulsátil, com pico nas primeiras horas de sono profundo e o controle é feito por feedback negativo. Fatores como o envelhecimento, a própria obesidade e estados hiperglicêmicos inibem sua secreção. Com o envelhecimento, os níveis séricos do GH vão sendo reduzidos gradativamente, de maneira que dos 5 aos 20 anos a concentração é $6 \mathrm{ng} / \mathrm{ml}$, já entre $40-70$ anos, apenas $1,6 \mathrm{ng} / \mathrm{ml}$. Essa redução de cerca de $25 \%$ causa déficit proteico nos tecidos e aumento da deposição de gordura (GUYTON AC e HALL JE, 2017).

Lubrano C, et al. (2020), apresenta evidências de que o déficit de GH decorrente do envelhecimento, além de proporcionar obesidade central, desregula o eixo imunológico de pacientes idosos, o que contribui diretamente para um pior prognóstico à Corona Virus Disease 2019 (COVID-19), doença que causa a atual pandemia. $\mathrm{O}$ GH reduz-se mais significativamente em homens que em mulheres, em todas as faixas etárias, e a gravidade da COVD-19 apresenta essa mesma tendência, ou seja, quanto maior o déficit de GH, maior a gravidade da doença, principalmente em homens. Assim, a reposição deste hormônio poderia trazer benefício para alguns grupos de pacientes, principalmente com a melhora da imunidade ao combate ao vírus.

O cortisol, principal hormônio glicocorticoide, é sintetizado no córtex da glândula adrenal e tem seu controle realizado por feedback negativo. Fatores como estresse físico ou mental, lesão tecidual, dor, entre outros, levam a sua produção e secreção. Picos secretórios ocorrem no início da manhã e final da noite, porém a secreção ocasionada pelo estresse ocorre independentemente do ciclo circadiano. O cortisol, entre outras funções, estimula a gliconeogênese, promove resistência à insulina pela menor utilização de glicose pelas células, reduz a síntese proteica levando a sarcopenia, aumenta a oxidação dos ácidos graxos e mobiliza-os a fim de gerar energia, contribuindo para a conservação de glicose e glicogênio corporais (GUYTON AC e HALL JE, 2017; GARBELLOTTO Gl, et al., 2018).

Freitas EV, et al. (2013), indica que a resposta do hormônio adrenocorticotrófico (ACTH) ao fator liberador de corticotrofina (CRF) está levemente aumentada no idoso, mas que o cortisol não se altera em decorrência do envelhecimento, apesar do aumento da camada fasciculada do córtex adrenal. Sabe-se que um dos principais fatores que aumenta a produção de cortisol é o estresse e, como a própria definição de envelhecimento já se estabelece menor capacidade do idoso lidar com estresse, pode-se afirmar que indiretamente a liberação de cortisol nessa fase está aumentada.

Faltam referências atuais para relacionar as alterações do cortisol a um público idoso, porém a relação entre obesidade e cortisol já está bem estabelecida na literatura. O cortisol, aumentado nos estados de estresse crônico, causa obesidade devido a maior velocidade de mobilização da gordura quando comparada à sua oxidação e também pela deposição excessiva sob forma de gordura visceral, isso aliado a condições de sedentarismo, má alimentação e excesso de ingestão calórica (GARBELLOTTO GI, et al., 2018).

A atividade da enzima 11-hidroxiesteroide desidrogenase sobre o tecido adiposo central está aumentada em obesos, logo, um distúrbio que desregule a quantidade de cortisol permite que essa enzima se eleve e tenha grande participação não só na obesidade central (visceral), como na Síndrome Metabólica (SM), na maioria dos índices antropométricos e na resistência insulínica, além de causar hiperfagia e aumentar o IMC. Portanto, a obesidade e o eixo hipotálamo-hipófise-adrenal (HHA) consistem em uma via de mão dupla, pois um desequilíbrio do eixo favorece a obesidade, assim como a obesidade perturba a regulação do eixo, que se encontra hiperresponsivo em obesos (PEREIRA AAFA, et al., 2018). 
Os hormônios sexuais Luteinizante (LH) e Foliculoestimulante (FSH) são produzidos na adeno-hipófise, recebem estímulos do hormônio hipotalâmico liberador de gonadotrofina $(\mathrm{GnRH})$, e agem no testículo e ovário para secreção de testosterona e estrogênio, respectivamente. A testosterona tem sua produção iniciada na adolescência e sofre redução a partir dos 50 anos, sendo considerado hipogonadismo quando os níveis são inferiores a $2 \mathrm{ng} / \mathrm{ml}$. Possui regulação por feedback negativo e, além de participar do desenvolvimento dos caracteres masculinos, aumenta a massa muscular e regula o acúmulo e distribuição de tecido adiposo (GUYTON AC e HALL JE, 2017).

A partir do fato de que o envelhecimento reduz os níveis de testosterona e que na literatura existe uma correlação negativa com a obesidade, de maneira que quanto menor o nível de testosterona, maior o IMC, conclui-se que idosos são mais propensos a serem obesos. Estudos indicam que essa redução hormonal faz aumentar a massa gorda e, também, que a privação androgênica propicia ganho de peso de 3,4kg e aumento da circunferência abdominal em 22\% em 6 meses. Em estados de hipogonadismo, a baixa testosterona circulante não consegue inibir a atividade da enzima lipase lipoproteica (LPL) do tecido adiposo e com isso haverá acúmulo de gordura no subcutâneo gerando obesidade (FUI MN, et al., 2014).

Os hormônios FSH e LH influenciam a produção e desenvolvimento dos folículos ovarianos a partir dos 912 anos na mulher, os quais, na ausência de fecundação, sofrem degeneração formando o corpo lúteo, o qual produz estrógeno. A partir da menopausa há o hipoestrogenismo com consequente alteração lipídica, aumento de peso e gordura abdominal que, aliado ao fato de mais da metade das mulheres não praticar exercícios físicos, reforça-se a teoria de que a prevalência de obesidade e sobrepeso nesse período também se relaciona ao estilo de vida, como má alimentação, sedentarismo e redução do gasto energético, com ganho médio de peso de 2-6kg no primeiro ano. Além disso, a grelina (hormônio da fome), está aumentada nessa fase (GUYTON AC e HALL JE, 2017; ZURITA RCM, et al., 2013; FCMSCSP, 2018).

Os hormônios tireoidianos, T3 e T4, são secretados pela glândula tireoide, que é controlada, principalmente, pelo TSH, produzido e secretado pela hipófise anterior sob estímulo do hormônio liberador de tireotropina (TRH), sintetizado por neurônios do hipotálamo, e tem como principal função o aumento generalizado da atividade funcional e metabólica dos tecidos corporais. Contudo, em pessoas que possuem o eixo hipotálamo-hipófise-tireoide intacto, a diminuição dos níveis sanguíneos de T3 e T4 exercem um feedback negativo, estimulando a liberação de TRH que estimula a secreção de TSH (GUYTON AC e HALL JE, 2017; BRENTA G, et al., 2013).

As alterações no eixo hipotálamo-hipófise-tireoide no processo de envelhecimento não são claras, porém estudos concluem que há elevação de TSH e dos títulos de autoanticorpos, assim como a resposta da glândula tireoide ao estímulo do TSH pode estar diminuída. Após os 60 anos há redução na taxa de captação de iodo pela tireoide levando à queda na produção dos hormônios tireoidianos que, muitas vezes, levam a características próprias da senescência. Com a exposição das células tireóideas ao estresse oxidativo ou resposta adaptativa relacionada ao envelhecimento, ocorre um prejuízo na estrutura e função tireoidiana que afeta tanto sua produção como ação (NEVES CMFP, et al., 2016; SILVA SM, et al., 2018).

Para a avaliação da função tireoidiana são indicadas a mensuração de TSH (com intervalo normal entre 0,45-4,5 mUI/L) e T4 livre, associado aos sinais e sintomas de disfunção. O hipotireoidismo subclínico (HSC) é caracterizado pelos níveis de TSH altos, com níveis de T4 livre normais. Como o TSH pode aumentar com a idade e não há um valor definido para o diagnóstico de HSC, não é vantajoso o rastreio em idosos pela dosagem desse hormônio, sendo necessária a determinação dos anticorpos anti-TPO e/ou anti-TG que, também, podem estimar a progressão para o hipotireoidismo clínico (HC), uma vez que essa ocorreu em 2,9\% em 5 anos de seguimento (FREITAS EV et al., 2013; NEVES CMFP, et al., 2016).

O HC tem impacto significativo no aumento de desenvolvimento de DCV, alterações do humor e cognitivas, diminuição da densidade mineral óssea e dislipidemia, porém com a reposição precoce de tiroxina, algumas condições podem se normalizar. Como no HSC tem redução dos hormônios T3 e T4, importantes no metabolismo das gorduras e carboidratos, o tratamento dessa condição não deve ser postergado evitando, assim, a obesidade (BRENTA G et al., 2013; NEVES CMFP, et al., 2016; SILVA SM et al., 2018). 
A secreção de insulina e glucagon acontece nas ilhotas de Langerhans do pâncreas, que contém células alfa $(\alpha)$, que secretam glucagon, e beta $(\beta)$ que secretam insulina. Esses hormônios são antagonistas fisiológicos com ação crucial para a regulação do metabolismo da glicose, lipídios e proteínas. Durante o período de alimentação, há aumento da secreção de insulina, a qual remove glicose sérica e estimula sua captação periférica (muscular, hepática, gordurosa), concomitantemente à diminuição dos níveis séricos de glucagon, que aumentam no jejum para manutenção dos níveis séricos de glicose, e inibição da secreção de insulina (GUYTON AC e HALL JE, 2017).

O tipo 2 corresponde a 95\% dos casos de Diabetes Melitus (DM) e está relacionada ao sobrepeso e obesidade, cada vez mais prevalentes no idoso. Santos RR, et al. (2013) afirma que a glicemia de jejum aumenta em 1-2 mg/dL e a glicemia pós-prandial em 10 a $20 \mathrm{mg} / \mathrm{dL}$ a cada década depois dos 30 anos de idade. O envelhecimento, isoladamente, não é o responsável pelo aumento da resistência insulínica, e sim a mudança na distribuição corporal que ocorre nesses indivíduos, já que a intolerância à glicose se associa à obesidade abdominal e à inatividade dos idosos (FREITAS EV, et al., 2013).

A grelina e a leptina são hormônios antagonistas, regulam a sensação de fome e saciedade, essenciais para um adequado suprimento alimentar. Esses sentimentos são influenciados por vias neurais do hipotálamo que regulam o comportamento alimentar através de um conjunto de reações químicas, hormonais e centrais que controlam a quantidade e qualidade nutricional dos alimentos ingeridos (GUYTON AC e HALL JE, 2017; MAIOR AS, 2012).

A grelina é um hormônio orexígeno liberado, principalmente, pelas células oxínticas do estômago e tem seus níveis sanguíneos aumentados durante o jejum. Já a leptina é um hormônio anorexígeno liberado pelos adipócitos proporcionalmente ao tecido adiposo, sinalizando excesso de armazenamento energético. A estimulação dos receptores leptínicos tem como objetivo aumentar o metabolismo e o gasto energético, agindo como intermediário entre tecido adiposo e cérebro quando energia suficiente foi armazenada e a ingestão de alimentos não é mais necessária. Além disso, a leptina é um agente protetor contra a adiposidade na busca pela homeostase energética (GUYTON AC e HALL JE, 2017).

O desequilíbrio do balanço energético e o aumento da adiposidade parecem contribuir com a resistência da ligação da leptina aos seus receptores hipotalâmicos. Em mutações que resultam na incapacidade das células adiposas produzirem leptina ou que geram receptores leptínicos defeituosos no hipotálamo, pode ocorrer hiperfagia acentuada e obesidade mórbida. Outra relação é quanto ao número de horas de sono por noite inversamente relacionado ao IMC e obesidade, e esta privação do sono produz hiperfagia pela diminuição da secreção de leptina e aumento dos níveis de grelina, aumentando a fome e o apetite e, consequentemente, a obesidade (ABESO, 2016; MAIOR AS, 2012; SANDE-LEE S e VELLOSO LA, 2012).

Pela Organização Mundial de Saúde (OMS), o excesso de peso é diagnosticado pelo IMC $\geq 25 \mathrm{~kg} / \mathrm{m}^{2}$, porém devido a mudanças fisiológicas na composição corporal dos idosos com o aumento progressivo da gordura visceral e perda progressiva de massa muscular, em consequência à sarcopenia, o Ministério da Saúde (MS) aceita que nessa população o IMC normal seja de $22-27 \mathrm{~kg} / \mathrm{m}^{2}$, sendo considerado sobrepeso e obesidade, respectivamente $27-29,9 \mathrm{~kg} / \mathrm{m}^{2}$ e $\geq 30 \mathrm{~kg} / \mathrm{m}^{2}$ (ABESO, 2016; HOLLANDA BC, et al., 2020).

O peso corporal tende a aumentar até média de 60 anos (o homem alcança seu ápice aos 65 e a mulher aos 75 anos) e, após, há diminuição do peso associado tanto à perda de massa muscular quanto da massa óssea (osteopenia e/ou osteoporose). Essas mudanças no envelhecimento tendem a mudar a composição e a distribuição de gordura corporal, com sua redução nos membros e acúmulo na região abdominal (JESUS AS e ROCHA SV, 2018; SANTOS RR, et al., 2013).

A promoção do envelhecimento saudável, bem como a importância da inclusão da avaliação da composição corporal e da força muscular devem ser rotina na atenção à pessoa idosa, principalmente porque o excesso de peso está relacionado diretamente à incapacidade funcional. A dificuldade na redução de peso com o aumento da idade se deve a alterações no metabolismo dos idosos, o que torna a obesidade uma condição crônica que predispõe o indivíduo a doenças cardiovasculares (DCV), principal causa de mortalidade em idosos, DM2, síndrome metabólica (SM), artrose, doença vascular periférica e alguns tipos de câncer. 
Apesar disso, a perda de peso em idosos é uma recomendação não muito simples, pois apesar de prevenir e tratar comorbidades, pode levar a perdas involuntárias de tecido ósseo e massa muscular, aumentando o risco de quedas e fraturas (SANTOS RR, et al., 2013; TESSER CD, 2017).

Mesmo sabendo que a obesidade causa piora na qualidade de vida dos idosos obesos quando comparado aos não obesos, sendo necessária a redução de peso nesses indivíduos, muitos profissionais ainda não recomendam o emagrecimento para esses pacientes devido à falta de estudos que mostram seus reais benefícios. Sabe-se que a redução intencional de peso em idosos obesos melhora o perfil de risco cardiovascular, reduz a inflamação crônica e, consequentemente, melhora a qualidade de vida. Porém, a perda de peso involuntária requer uma avaliação clínica cuidadosa, pois ela pode decorrer de alterações na distribuição corporal próprias do envelhecimento que levam à redução de peso em estágios tardios da vida, como pode ocorrer em consequência de processos patológicos (BEZERRA DS, et al., 2017; LIMA LM, et al., 2017; ALBUQUERQUE ALT, 2016).

Nesse contexto, a prevenção quaternária apresenta um foco relevante na atenção à saúde da população, que pode fazer uso de medicamentos em excesso. Assim, reduz-se a necessidade de intervenções médicas invasivas, diminuindo riscos de adoecimento, como a obesidade, sobretudo em estratégia de alto risco, devido ao seu grande potencial iatrogênico e impacto na morbimortalidade coletiva (TESSER CD, 2017; TESSER, CD, 2020).

\section{CONSIDERAÇÕES FINAIS}

O processo fisiológico de envelhecer, conhecido por senescência, traz uma série de mudanças corporais em relação ao tecido muscular e gordura, e na produção e liberação de vários hormônios do metabolismo, facilitado a obesidade. Os hormônios reduzidos no envelhecimento são: GH, estrógeno, testosterona, T3, T4, leptina e insulina. Já o TSH, cortisol e grelina elevam. O aumento da gordura corporal reduz a funcionalidade e aumenta o risco para DCV, distúrbios musculoesqueléticos, câncer e mortalidade, assim, discutir sobre as alterações endócrino-metabólicas na senescência torna-se mais relevante, uma vez que facilitam a obesidade. Ressalta-se que mudanças nos hábitos de vida que levam à diminuição do gasto energético influenciam no ganho de peso. Por fim, destaca-se a escassez de publicações atuais sobre fisiologia endócrina no idoso, uma vez que os postulados da endocrinologia são imutáveis ao longo das décadas.

\section{REFERÊNCIAS}

1. ALBUQUERQUE ALT, et al. Atualização das diretrizes em cardiogeriatria da sociedade brasileira de cardiologia. Arq Bras Cardiol, 2019; 112(5): 649-705.

2. ASSOCIAÇÃO BRASILEIRA PARA ESTUDO DE OBESIDADE E DA SÍNDROME METABÓLICA. Diretrizes Brasileiras de Obesidade. São Paulo: ABESO; 2016; 188p. Disponível em: https://abeso.org.br/wpcontent/uploads/2019/12/Diretrizes-Download-Diretrizes-Brasileiras-de-Obesidade-2016.pdf. Acesso em: 08 set 2020.

3. BEZERRA DS, et al. Obesidade, síndrome metabólica e impacto na saúde do idoso brasileiro. Rev Nova Esperança, 2019; 17(1): 71-78.

4. BRASIL. Instituto Brasileiro de Geografia e Estatística (IBGE). Pirâmide etária. Brasil, 2020. Disponível em: https://educa.ibge.gov.br/jovens/conheca-o-brasil/populacao/18318-piramide-etaria.html. Acesso em: 04 jun 2020.

5. BRENTA G, et al. Diretrizes clínicas práticas para o manejo do hipotireoidismo. Arq Bras Endocrinol Metab, $2013 ; 57$ (4): 265-99.

6. FREITAS EV, et. al. Tratado de Geriatria e Gerontologia. 3nd ed. Rio de Janeiro: Guanabara Koogan, 2013; 1750p.

7. FUI MN, et al. Lowered testosterone in male obesity: mechanisms, morbidity and management. Asian journal of andrology, 2014, 16(2).

8. GARBELLOTTO Gl, et al. Associação do cortisol salivar com os componentes da síndrome metabólica. ABCD, arq. bras. cir. dig., São Paulo, 2018, 31: e1351.

9. GUYTON AC, HALL JE. Tratado de Fisiologia Médica. 13a ed. Rio de Janeiro: Elsevier, 2017; 1150p.

10. HOLLANDA BC, et al. Impacto da obesidade sarcopênica na capacidade funcional de idosos. Rev Enferm UFPE on line, 2020; 14: e244093.

11. JESUS AS, ROCHA SV. Comportamento sedentário como critério discriminador do excesso de peso corporal em idosos. Rev Bras Ativ Fís Saúde, 2018; 23: e0030. 
12. LIMA LM, et al. Prevalência de sobrepeso e obesidade em idosas de centro de convivência para a terceira idade de Vitória/ES. Revista Brasileira de Ciências da Saúde, 2017; 21(2): 119-126.

13. LOPES AC. Tratado de Clínica Médica. 3a edição, Rio de Janeiro: Guanabara Koogan, 2016; 6148p.

14. LUBRANO C, et al. Is Growth Hormone Insufficiency the Missing Link Between Obesity, Male Gender, Age, and Covi19 Severity? Obesity, 2020; 28 (11): 2038-2039.

15. MAIOR AS. Regulação hormonal da ingestão alimentar: um breve relato de caso. Medicina (Ribeirão Preto). 2012; 45(3): 303-9.

16. NEVES CMFP, et al. Hipotireoidismo subclínico em idosos na atenção primária: ênfase na prevenção quaternária. Revista.hupe.uerj.br, 2016; 15(3).

17. PEREIRA AAFA, et al. Relação entre o hormônio cortisol e a síndrome metabólica. Salvador. Revista UNIFACS, 2018, 17.

18. MENOPAUSA E AUMENTO DE PESO. 2018. In Faculdade de Ciências Médicas da Santa Casa de São Paulo (FCMSCSP). São Paulo: Fundação Vieira de Carvalho. Disponível em: https://fcmsantacasasp.edu.br/menopausa-eaumento-de-peso/. Acesso em: 05 jun. 2020.

19. SANDE-LEE SV, VELLOSO LA. Disfunção hipotalâmica na obesidade. Arq Bras Endocrinol Metab, 2012; 56(6): 34150.

20. SANTOS RR, et al. Obesidade em idosos. Rev Med Minas Gerais, 2013; 23(1): 64-73.

21. SGARBIERI VC, PACHECO MTB. Healthy human aging: intrinsic and environmental factors. Braz. J. Food Technol., Campinas, 2017, 20: e2017007.

22. SILVA SM, et al. Hipotiroidismo subclínico no idoso. Acta Med Port, 2018; 31(12): 766-773.

23. SENESCÊNCIA E SENILIDADE - QUAL A DIFERENÇA? 2016. In: SOCIEDADE BRASILEIRA DE GERIATRIA E GERONTOLOGIA. São Paulo: SBGG. Disponível em: http://www.sbgg-sp.com.br/pub/senescencia-e-senilidade-quala-diferenca/. Acesso em: 04 jun 2020.

24. TESSER CD. Por que é importante a prevenção quaternária na prevenção? Rev Saude Publica, 2017; 51:116.

25. TESSER CD. Convergências entre prevenção quaternária e promoção da saúde. Rev Med Fam Comunidade, 2020; 15(42): 25-15.

26. ZURITA RCM, et al. Sobrepeso e obesidade em mulheres no climatério em um município do noroeste do estado do Paraná. Colloquium Vitae, 2013, 5: 47-55. 